The University of Maine

\title{
DigitalCommons@UMaine
}

Maine-Syracuse Longitudinal Papers

Maine-Syracuse Longitudinal Study

Fall 11-9-2016

\section{Challenging the "jolly fat" hypothesis among older adults: High body mass index predicts increases in depressive symptoms over a 5-year period}

Peter J. Dearborn MA

University of Maine, peterdearborn@gmail.com

Michael A. Robbins Ph.D.

University of Maine, michael_robbins@umit.maine.edu

Merrill F. Elias Ph.D., MPH

University of Maine,mfelias@maine.edu

Follow this and additional works at: https://digitalcommons.library.umaine.edu/ longitudinal_papers

Part of the Health Psychology Commons

\section{Repository Citation}

Dearborn, P.J., Robbins, M.A. \& Elias, M.F. (2016) Challenging the "jolly fat" hypothesis among older adults: High body mass index predicts increases in depressive symptoms over a 5-year period. Journal of Health Psychology 21, 1-11. doi: 10.1177/

1359105316675209 
Challenging the "jolly fat" hypothesis among older adults: High body mass index predicts increases in depressive symptoms over a 5-year period

\section{Abstract}

5 Several investigators have observed lowered risk for depression among obese older adults,

6 coining the "jolly fat" hypothesis. We examined this hypothesis using baseline and 5-year

7 follow-up BMI, depressive symptoms, and covariates from 638 community-based older adults.

8 High objectively measured BMI and functional limitations predicted increased future depressive 9 symptoms. However, symptoms did not predict future BMI. Self-reported BMI showed similar associations despite underestimating obesity prevalence. Results did not differ on the basis of

11 gender. Results for this study, the first longitudinal reciprocal risk analysis between objectively

12 measured BMI and depressive symptoms among older adults, do not support the "jolly fat"

13 hypothesis. 
1

Recent meta-analytic evidence suggests the existence of a relationship between obesity and depression (de Wit et al. 2010). However, the directional nature of this relationship remains less clear among differently aged and gendered samples. Among longitudinal studies, there is some evidence that depression status predicts later obesity. In a meta-analysis of 17 samples, Blaine (2008) estimated a large effect size of baseline depression status on subsequent obesity when controlling for baseline obesity and other covariates. However, the only study composed entirely of older adults (age > 50; DiPietro et al. 1992) found that baseline depression was associated with marginally lower risk for future obesity, results consistent with the "jolly fat" hypothesis which posits that there are protective effects of obesity on risk for depressive symptoms (Crisp \& McGuiness 1976; Crisp et al. 1980; Palinkas et al. 1996). Results supportive of the "jolly fat" hypothesis have primarily been observed for older cohorts, have sometimes been stronger for males than for females, and have been obtained mostly from cross-sectional studies. Indeed, a major conclusion from a systematic review of epidemiological research is that there is a dearth of high quality longitudinal studies which measure the effect of obesity on future depression status (Atlantis \& Baker 2008). Of the four studies which met their quality inclusion standards, three were composed of older adults, all from the same sample in Alameda County, California (Roberts et al. 2003; Roberts et al. 2000; Roberts et al. 2002). Contrary to the "jolly fat" hypothesis, both one and five-year longitudinal analysis revealed that self-reported obesity predicted significantly higher risk for depressive symptoms even with control for an array of covariates. These results have been supported by more recent longitudinal analysis of older adults, though findings vary by obesity measurement used. While studies using self-reported BMI have found obesity increasing risk for depressive 
1 symptoms among women (Pan et al. 2012), but not in men (Xiang \& An 2015), a study using objectively measured BMI found this association only among men (Vogelzangs et al. 2010). To our knowledge, only two longitudinal studies have systematically assessed the reciprocal effects of depression and obesity among older adults (Roberts et al. 2003; Pan et al. 2012). Both studies found that obese individuals were at a higher risk for reporting clinical levels of depressive symptoms, anti-depressant use, or receiving a diagnosis of depression 5-10 years later. This was true even with control for baseline depression status and a range of covariates. Conversely, although Pan et al. (2012) found evidence of a bidirectional relationship, Roberts et al. (2003) did not find that depressed individuals were at a higher risk for obesity five years later. Compared with prior longitudinal studies on depression and obesity, the covariate models employed by Roberts et al. and Pan et al. were considerably more extensive.

Although these studies have methodological strengths, both were also limited to using self-reported obesity. Prior studies have found systematic differences between objectively measured $\left(\mathrm{BMI}_{\mathrm{O})}\right.$ and self-reported $\mathrm{BMI}\left(\mathrm{BMI}_{\mathrm{SR}}\right)$. Specifically, individuals tend to overestimate height and underestimate weight, which results in a likely underestimation of obesity prevalence, particularly among older adults (Kuczmarski et al. 2001; Keith et al. 2011). Additionally, Keith et al. (2011) noted that $\mathrm{BMI}_{\mathrm{SR}}$ failed to consistently predict mortality outcomes which were associated with $\mathrm{BMI}_{\mathrm{O}}$. As such, it is possible that $\mathrm{BMI}_{\mathrm{SR}}$ and $\mathrm{BMI}_{\mathrm{O}}$ may associate differently with other psychobiological outcomes.

The present study uses objective measurements of BMI from the Maine-Syracuse Longitudinal Study (MSLS) to investigate the reciprocal effects of depressive symptoms and BMI and to further test the jolly-fat hypothesis among older adults. We hypothesize that BMI 
1 will be positively associated with concurrent and future depressive symptoms; i.e., will be seen

2 with cross-sectional and longitudinal analyses.

\section{Methods}

$4 \quad$ Participant Selection

The MSLS includes 7 waves of demographic, cardiovascular risk factor and cognitive performance data gathered over a period of 35 years employing a sample of community-dwelling adults residing in Central New York. Since the original MSLS baseline [1976], individuals have

8 been excluded based on diagnosed alcoholism, diagnosed psychiatric disorder, or inability to

9 speak English. In this and other subsequent MSLS studies, additional exclusionary criteria have

10 been included on a study-by-study basis. The University of Maine Institutional Review Board

11 approved this study (reference number: 2005-07-04) and informed consent was obtained from all 12 participants.

13 Attrition

At baseline, older adults were selected for this analysis $(n=893, \geq 50 \mathrm{y})$. Participants no

15 longer residing in New York State were lost to the study due to changes in research funding

16 limiting travel of the investigative team out of state. Of the baseline participants, $71.4 \%$

17 responded again and were eligible at wave 7 follow-up $(n=638)$. Additionally, participants

18 were excluded from regression analysis for missing depressive symptom data $(n=27)$, for

19 missing objectively measured $\left(\mathrm{BMI}_{\mathrm{O}} ; n=42\right)$ or self-reported $\mathrm{BMI}\left(\mathrm{BMI}_{\mathrm{SR}} ; n=117\right)$, or baseline 20 covariate data $(n=25)$.

Compared with individuals who were lost to follow-up or excluded, individuals retained

22 at follow-up were 2.52 years younger (CI: $1.04-4.00, p=.002), 2.5$-points lower on the Zung

23 Depression Inventory (ZDI; CI: 1.06 - 3.95, $p=.001$ ), and had 0.48 fewer physical deficits (CI: 
$10.30-0.66, p<.001)$ at baseline. However, neither group differed in baseline educational level,

2 marital status, number of recent life changes, social activity/isolation level, number of chronic

3 illnesses, or $\mathrm{BMI}_{\mathrm{SR} / \mathrm{O}}(p>.05)$. Table 1 presents baseline descriptive statistics for participants

4 retained at follow-up.

5 INSERT TABLE 1 HERE

\section{Study Design}

The present study uses BMI, depressive symptoms, and covariate information from wave

86 of the MSLS [2001-2005] as baseline data. Approximately 5-years later [2006-2010] at wave

97 follow up, BMI and depressive symptoms were obtained. Objective measurement of height,

10 weight, and physical performance obtained at wave 6 and wave 7 allowed for more

11 comprehensive prospective models than were available in previous waves.

\section{Measures of Obesity and Depressive Symptoms}

Objectively measured height and weight at baseline and follow-up was assessed in the lab

14 without shoes or heavy clothing to calculate BMI $\left(\mathrm{kg} / \mathrm{m}^{2}\right)$. In addition, self-reported height and

15 weight were used to calculate an alternative index of BMI. BMI was analyzed as a continuous

16 outcome and baseline adjustment variable and obesity was defined based on the World Health

17 Organization obesity standard (BMI $\geq 30 ;$ WHO, 2010).

Baseline and outcome depressive symptoms were measured with the ZDI (Zung, 1965).

The ZDI is composed of 20 self-reported, Likert scale items ranging in frequency from 1 "a little

20 of the time" to 4 "most of the time" and summed to create a total raw score. Raw scores were

21 then divided by 0.8, resulting in an index score range from 25 to 100 (Hunter \& Murphy 2011).

22 At baseline, ZDI items showed good internal consistency $(\alpha=.80)$. The ZDI has been reported

23 to lack sufficient sensitivity to act as a categorical diagnostic measure (Thurber et al. 2002). As 
1 such, index scores were used to represent the continuum of depressive symptom severity rather

2 than major depressive disorder incidence. Individuals missing more than 2 items were excluded

3 from analysis, scores for 2 or fewer missing items were imputed based on the mean of available

4 ZDI data.

\section{Covariates}

Covariates were selected based primarily on the extensive models used by Roberts et al.

7 (2003), their theoretical relevance as posited by Atlantis and Baker (2008), and their associations

8 with BMI and ZDI within our own sample. This included adjustments for age, gender, education

9 in years, marital status (married/living w/ partner vs. other), social isolation (no close friendships

10 and/or co-residents vs. other), social activity (see: Robbins et al. 1994), recent life events

11 (yes/no), physical functioning, and chronic medical conditions.

For the chronic illness composite, participants completed questionnaire items asking

13 whether their doctor had told them if they had a range of medical issues during the past 6 years.

14 This included summing occurrence data on heart trouble, high blood pressure, asthma,

15 bronchitis/emphysema, rheumatoid arthritis, diabetes, stroke, cancer, cataracts, and circulatory

16 issues.

17 The physical functioning deficit composite was measured with 3 timed performance

18 tasks. Participants were asked to 1) stand up 3 times from a hard-backed chair with their arms

19 folded, 2) walk forward ten feet, turn $180^{\circ}$, and walk back to their starting position, 3) from a

20 standing position, complete a full $360^{\circ}$ turn. Participants unable to complete a task or whose task

21 completion time was among the slowest quartile in the baseline sample were considered to have

22 a deficit in that area of performance. Total deficit scores ranged from "0" (no deficit) to " 3 " 
1 (high deficit). Prior research has shown that a performance deficit in any of these tasks is

2 predictive of future functional dependence in activities of daily living (Gill et al. 1995). Self-reported family income, smoking status, and ethnicity were all collected and

4 considered for analysis, but ultimately dropped from our final models. Family income was

5 considered because it has been found to be related to depressive symptoms (Mendes De Leon et

6 al. 1994). However, it was dropped from analysis because 1) a large number $(n=106)$ of

7 participants indicated that they "would rather not answer" this item and 2) when tested as a

8 model covariate, family income did not change the results reported below. Smoking status was

9 considered based on the quality assessment criteria by Atlantis and Baker (2008), but was

10 dropped due to a lack of observed association with $\mathrm{BMI}_{\mathrm{SR} / \mathrm{O}}$ or ZDI scores. Although ethnicity

11 may moderate the relationship between obesity and depressive symptoms (Xiang \& An 2015),

12 detailed group analysis was not conducted due to a lack of sufficient sample size for non-white

13 participant groups (Black/Hispanic/Asian/American Indian combined; $n=42$ ).

\section{Statistical Analysis}

Hierarchical multivariate regression models were used to explore the cross-sectional and

16 longitudinal association between BMI and ZDI with adjustment for covariates. Baseline (wave

17 6) values for ZDI and BMI, respectively, were used as covariates for models in which wave 7

18 ZDI or wave 7 BMI was the outcome of interest. Model 1 of the cross-sectional and longitudinal

19 analyses included baseline age, sex, education, and marital status as covariates. Model 2

20 contained all covariates from Model 1 in addition to life events, social isolation, and social

21 activity. Model 3 included all covariates from Model 2 in addition to chronic health conditions

22 and physical performance deficits. 
Model variables met assumptions for parametric multivariate regression (Durbin-Watson; $1.94 \geq d \leq 2.04$ ) and minimal collinearity was observed (Max. variance inflation factor $\leq 1.97$ ).

Tests for non-linear associations between BMI and ZDI were conducted with the addition of

4 quadratic predictor terms with adjustment for linear terms. Neither inclusion of quadratic terms

5 for wave $6 \mathrm{BMI}_{\mathrm{SR} / \mathrm{O}}(p>.25)$ nor wave $6 \mathrm{ZDI}(\mathrm{p} \geq .09)$ improved fit across all models.

Statistical analyses were conducted with SPSS software (version 23.0).

\section{Results}

\section{Objective vs. Self-Reported BMI}

Bivariate and paired-samples t-test analysis were used to examine the convergent validity

between objective and self-reported height and weight at baseline for participants who completed

11 both wave 6 and wave 7 . Participants both significantly underestimated their weight $[t(601)=-$

$1210.71, p<.001]$ by an average of $2.10 \mathrm{~kg}(95 \% \mathrm{CI}=1.72 \mathrm{~kg}, 2.48 \mathrm{~kg})$ and overestimated their

13 height $[t(574)=6.02, p<.001]$ by an average of $.94 \mathrm{~cm}(95 \% \mathrm{CI}=.63 \mathrm{~cm}, 1.25 \mathrm{~cm})$. However,

14 self-reported and objective measurement of height $(r=.93, p<.001)$ and weight $(r=.97, p<$

$15.001)$ were also highly associated. BMI $_{\text {SR }}$ obesity prevalence (29.2\%) was significantly lower

16 than what would be expected $\left[\chi^{2}(1,572)=348.57, p<.001\right)$ based on the $\mathrm{BMI}_{\mathrm{O}}$ obesity

17 prevalence in our sample $(38.8 \%)$.

18 Model Analyses

Results of analyses for cross-sectional and longitudinal multivariate regression models

20 are presented in Table 2. With regard to the cross-sectional association between baseline BMI

21 and ZDI, across all models the adjusted $R^{2}$ values ranged from .07 to .20 . $\mathrm{BMI}_{\mathrm{O}}$ predicted higher

22 ZDI in Model1 and Model $2(p$ 's $<.01)$, but not in Model $3(p=.16)$. BMI SR predicted higher

23 ZDI in Model $1(p<.01)$, but not in Model 2 or Model $3(p$ 's $>.10)$. 
Regression models examining the longitudinal association between baseline BMI and 5year follow-up ZDI included adjustment for baseline ZDI as well as the other covariates. Across all models, adjusted $R^{2}$ values ranged from .44 to .46 . Baseline ZDI was strongly correlated with follow-up ZDI $(r=.65, p<.001)$, accounting for $26.6 \%$ to $35.6 \%$ of the total variance across all models. Both higher $\mathrm{BMI}_{\mathrm{O}}$ and $\mathrm{BMI}_{\mathrm{SR}}$ at baseline predicted higher $\mathrm{ZDI}$ at follow-up across all three regression models $(p$ 's $<.001)$.

In addition to BMI, the covariates age and physical functioning were significant predictors of follow-up ZDI. For instance, in the $\mathrm{BMI}_{\mathrm{O}}$ models: 1) older age was associated with higher follow-up ZDI in Model $1(b=.07, S E=.03, p=.029)$ and in Model $2(b=.07, S E=.03$, $p=.023)$, but not when adjusting for physical ailments and physical functioning in Model $3(p>$ $.25)$; and 2) baseline physical functioning deficits predicted higher follow-up ZDI $(b=.95, S E=$ $.29, p=.001)$ in the full regression model (Model 3).

Regression models examining the longitudinal association between baseline ZDI and follow-up BMI included adjustment for baseline BMI and the other covariates. Across all models, adjusted $R^{2}$ values ranged from .68 to .78 . Baseline BMI was highly correlated with follow-up BMI $\left(r_{\text {objective }}=.89, r_{\mathrm{SR}}=.83\right)$, accounting for $56.0 \%$ to $67.7 \%$ of the total variance across all models. For both BMI variables across all three models baseline ZDI failed to predict follow-up BMI $(b \leq .02, p \geq .20)$.

INSERT TABLE 2 HERE

Supplementary analyses were conducted to examine gender differences, given that evidence supporting the "jolly fat" hypothesis has been found predominantly for males, that is, others have found that obese men and women have differential levels of risk for depressive symptoms (Atlantis \& Baker 2008; Xiang \& An 2015). Adjusted for all other cross-sectional 
1 (wave 6) model 3 covariates, females reported higher ZDI scores than males (in $\mathrm{BMI}_{\mathrm{O}}$ model, $b$

$2=2.18, S E=.66$; in $\mathrm{BMI}_{\mathrm{SR}}$ model, $b=2.16, S E=.68, p$ 's $\left.<.01\right)$. However, results for

$3 \mathrm{BMI}^{*}$ gender interaction terms included in the $\mathrm{BMI}_{\mathrm{SR} / \mathrm{O}}$ cross-sectional and longitudinal models

4 were non-significant ( $p$ 's $>.25$ ) when predicting either baseline ZDI or longitudinal change in 5 ZDI.

\section{Discussion}

In this longitudinal study, we tested the "jolly fat" hypothesis in the context of assessing

8 the relationship between depressive symptoms and BMI among a community sample of older

9 adult men and women. We found that older adults with higher baseline BMI reported increases

10 in depressive symptoms over a five-year period. This was true even when adjusting for baseline

11 depressive symptoms, age, sex, education, marital status, social isolation, social activity, chronic

12 illness, and physical functioning. These results were unchanged whether self-reported or

13 objectively measured BMI was used, thus validating the self-report findings of Roberts et al.

14 (2003).

\section{Strengths}

This study adds methodological strength to the currently limited longitudinal research on

17 obesity and depression among older adults, much of which is lacking objective measurement of

18 BMI (Atlantis \& Baker 2008). Although we found in our sample that objective measurement

19 and self-reported height and weight were highly correlated and similar in predicting baseline

20 level and change in depressive symptoms, there was also a small, but consistent tendency for

21 participants to underestimate weight while overestimating height, resulting in a considerable

22 underestimation of the true obesity prevalence within our sample. While using self-reported

23 BMI as a continuous variable is likely justifiable for associative analysis, logit models, in which 
1 dependent variables are categorical (i.e., self-reported obesity; Cox 1958) may experience a

2 considerable loss of predictive power due to misclassification. Additionally, researchers

3 studying obesity prevalence are encouraged to either use objective BMI or to consider statistical

4 adjustments for reporting bias among older adults.

Regression models also incorporated a composite measure of physical functioning which

6 consisted of objectively timed performance tasks predictive of difficulty in activities of daily

7 living (Gill et al. 1995), which up until this point has been missing from associative research

8 examining depression and obesity. Additionally, although Roberts et al. (2003) adjusted for self-

9 reported functional limitations (e.g., bathing, dressing), they did not include objective

measurement of physical functioning. Prior studies have reported moderate overlap, but distinct

11 differences between self-reported and objectively measured physical functioning. Until now,

12 studies have been unable to account for the potentially confounding associations between self-

13 reported physical functioning and well-known dimensions of depression such as self-efficacy

14 and mastery rather than actual performance (Becofsky et al. 2013; Kempen et al. 1996).

Lastly, in this study we observed changes in BMI and depressive symptoms over time by

16 including both baseline and follow-up levels in our models. This allowed us to simultaneously

17 test the bidirectional associations between BMI and depressive symptoms among older adults,

18 adding to the scant research with such a design (Pan et al. 2012; Roberts et al. 2003). Although

19 there is evidence for symptoms of depression being both an outcome and predictor of incident

20 obesity (Blaine 2008; Atlantis \& Baker 2008), to our knowledge no meta-analysis has

21 statistically accounted for potential publication bias. As such, the strength of pooled evidence

22 for bidrectionality as assessed by non-reciprocal models may be overestimated. In the context of 
1 a reciprocal analysis and a sufficiently powered sample, we observed significant directional

2

associations with BMI as predictor, but not as an outcome of baseline depressive symptoms.

\section{Limitations}

It should be noted that our sample was both highly educated and predominately white. This limited our ability to conduct a more thorough analysis of socioeconomic and ethnic group differences which may moderate the association between obesity and depressive symptoms (Xiang \& An 2015). However, with regard to our main predictor/outcome variables, obesity and depressive symptoms, our sample did not vary considerably from national estimates among older adults (Center for Behavioral Health Statistics and Quality 2015; Ogden et al. 2014). For additional health demographic information of the MSLS sample see Crichton et al. (2014).

Regarding attrition, participants who were available at follow-up tended to be younger, less likely to report depressive symptoms, and less functionally impaired compared with those who were unavailable. Although functional impairment and to a lesser extent advanced age were associated with increased depressive symptoms in our models, it is possible that the magnitude of their effects were partially attenuated. Similarly, the effect of depressive symptoms on BMI may have been partially attenuated; however, there was little evidence for a bidirectional trend.

Our results indicate that higher BMI may contribute to increases in depressive symptoms over time. Despite our findings, it is possible that chronic illness and low physical functioning, which tend to increase risk for depressive symptoms may contribute to weight loss among aging adults as well as weight gain due to lowered physical activity and loss of muscle tone (StArnaud-McKenzie et al. 2010). However, only one individual in our wave 6 sample was measured to be underweight by objective measurement $(\mathrm{BMI}<18.5)$. As such, our conclusions are limited to normal and overweight/obese older adults and our results may mask associations 
1 between low-weight and depressive symptoms mediated by nutrient deficits as a result of

2 appetite loss (i.e., B12, folate; Kim et al. 2008). However, our results are consistent with the

3 homeostasis theory of obesity which posits that some individuals may over-consume energy-

4 dense, low-nutrient foods (e.g, sugar-sweetened beverages) as a form of emotion regulation

5 (Marks 2016). These homeostatic disturbances extended over the course of several years may

6 contribute to continued weight gain and declines in physical health which in turn increases risk

7 for experiencing depressive symptoms.

8 Conclusions and Future Directions

9 The results of this study clearly do not support the "jolly fat" hypothesis among older

adults. This is an important finding because the notion that obesity is protective may lead to

11 behaviors that are not consistent with good health practices. Higher BMI was related to greater

12 risk, not less risk, for depressive symptoms. There was no indication that the BMI-associated

13 risk for depressive symptoms interacted with gender. Studies that obtained results supportive of

14 the "jolly fat" hypothesis among older adults, and predominantly for males, were conducted two

15 or more decades ago (Crisp \& McGuiness 1976; Crisp et al. 1980; Palinkas et al. 1996; DiPietro

16 et al. 1992). It is possible that increased awareness of the diversity and severity of health risks

17 associated with obesity has made positive associations with depressive symptoms more likely not

18 only through resultant individual perceptions of threat to health but also through an increased

19 prevalence of perceived weight-based discrimination overall among U.S. adults (Andreyeva et al.

20 2008). Although women likely experience greater weight-related discrimination overall

21 compared with men, more recent analysis suggests that these differences may not extend into

22 older adulthood (Roehling et al. 2007; Eichner 2002; Puhl et al. 2008). Lastly, recent findings do

23 suggest that older adults and especially the oldest-old ( $\geq 80 \mathrm{y})$ experience considerably lower 
1 rates of weight-based discrimination compared with younger adults (Puhl et al. 2008; Jackson et

2 al. 2015), which may explain previously inconsistent findings between younger and older

3 samples.

$4 \quad$ Conversely, we did not find any association between baseline depressive symptoms and

5 BMI change five years later, contrary to the 10-year bidirectional results reported by Pan et al.

6 (2012). It is possible that behaviors associated with depressive symptoms (e.g., appetite

7 disturbance, lowered activity) require a longer incubation period in order to cause significant

8 weight gain among older adults. In our own 5-year models, BMI was measured to be highly

9 stable, which may have masked the potential for more subtle weight gain over a longer duration.

Regardless of gender, higher baseline BMI was associated with a net increase of

11 depressive symptoms. Although this latter finding is similar to the longitudinal results of

12 Roberts et al. (2003), it differs from other cross-sectional and longitudinal results that have

13 shown obese women to be at a higher risk for depressive symptoms in comparison to men

14 (Atlantis \& Baker 2008; Xiang \& An 2015). Our results indicate that although older adult

15 women may be at a higher risk for depressive symptoms, their risk for depressive symptoms as a

16 function of BMI does not differ significantly from that of older adult men. It is possible that

17 gender differences in weight-based discrimination diminish in older adulthood or that other

18 forms, such as age-based discrimination become more salient (Puhl et al. 2008). Future research

19 should investigate the potential mediating role of weight-based discrimination among obese

20 older adults in the development of depressive symptoms. In a review recently published by

21 Marks (2015), only one state (i.e., Michigan) was identified as having laws prohibiting weight

22 discrimination. However, this also opens up the possibility of tracking the efficacy of such 
1 legislation on reducing the prevalence of weight-based discrimination and incident depression

2 among overweight/obese individuals.

Objective physical impairment also independently predicted changes in depressive

4 symptoms and its combined association with chronic illness attenuated much of the association

5 between depression and aging. Future analysis should explore the possible mediating effect of

6 physical functioning interventions on the relationship between obesity and depressive symptoms.

7 It is possible that older obese individuals, who are more likely to experience depressive

8 symptoms may benefit from interventions targeting limitations in physical functioning.

9 Increased physical activity, which has been shown to reduce the risk for depressive symptoms among obese individuals (King et al. 2013), may play a key role by slowing the rate of functional

11 impairment independent of weight loss (Chalé-Rush et al. 2010).

\section{Supplementary Information Requests}

Additional information relating to this article may be obtained by request by emailing the primary author.

\section{Conflicts of Interest}

The authors declare that there are no competing financial interests in relation to the work described.

\section{References}

Andreyeva, T., Puhl, R.M. \& Brownell, K.D., 2008. Changes in perceived weight discrimination among Americans, 1995-1996 through 2004-2006. Obesity (Silver Spring, Md.), 16(5), pp.1129-1134.

Atlantis, E. \& Baker, M., 2008. Obesity effects on depression: systematic review of epidemiological studies. International journal of obesity (2005), 32(6), pp.881-891.

Becofsky, K., Baruth, M. \& Wilcox, S., 2013. Physical functioning, perceived disability, and depressive symptoms in adults with arthritis. Arthritis, 2013, p.525761. Available at: 
http://www.pubmedcentral.nih.gov/articlerender.fcgi?artid=3777208\&tool=pmcentrez\&ren dertype $=$ abstract.

Blaine, B., 2008. Does depression cause obesity?: A meta-analysis of longitudinal studies of depression and weight control. Journal of health psychology, 13(8), pp.1190-1197.

Center for Behavioral Health Statistics and Quality, 2015. Behavioral Health Trends in the United States: Results from the 2014 National Survey on Drug Use and Health, Available at: http://www.samhsa.gov/data/sites/default/files/NSDUH-FRR1-2014/NSDUH-FRR12014.htm.

Chalé-Rush, A. et al., 2010. Relationship between physical functioning and physical activity in the Lifestyle Interventions and Independence for Elders Pilot (LIFE-P). Journal of the American Geriatrics Society, 58(10), pp.1918-1924. Available at: http://www.ncbi.nlm.nih.gov/pmc/articles/PMC2952066/.

Cox, D., 1958. The regression analysis of binary sequences (with discussion). Journal of Royal Statistical Society Series B, 20, pp.215-242.

Crichton, G.E. et al., 2014. Cardiovascular health and cognitive function: the Maine-Syracuse Longitudinal Study. PloS one, 9(3), p.e89317. Available at: http://www.ncbi.nlm.nih.gov/pubmed/24595096 [Accessed July 26, 2016].

Crisp, A.H. et al., 1980. "Jolly fat” revisited. Journal of Psychosomatic Research, 24(5), pp.233241. Available at: http://www.sciencedirect.com/science/article/pii/0022399980900136.

Crisp, A.H. \& McGuiness, B., 1976. Jolly fat: relation between obesity and psychoneurosis in general population. British Medical Journal, 1(6000), pp.7-9. Available at: http://www.ncbi.nlm.nih.gov/pmc/articles/PMC1638245/.

DiPietro, L. et al., 1992. Depressive symptoms and weight change in a national cohort of adults. International journal of obesity, 16(10), pp.745-753.

Eichner, J.E., 2002. Apolipoprotein E Polymorphism and Cardiovascular Disease: A HuGE Review. American Journal of Epidemiology, 155(6), pp.487-495. Available at: http://aje.oxfordjournals.org/content/155/6/487.full [Accessed March 23, 2016].

Gill, T.M., Williams, C.S. \& Tinetti, M.E., 1995. Assessing risk for the onset of functional dependence among older adults: the role of physical performance. Journal of the American Geriatrics Society, 43(6), pp.603-609.

Hunter, E.E. \& Murphy, M.M., 2011. Zung Self-Rating Depression Scale. In J. Kreutzer, J. DeLuca, \& B. Caplan, eds. Encyclopedia of Clinical Neuropsychology. Springer New York, pp. 2746-2747. Available at: http://dx.doi.org/10.1007/978-0-387-79948-3_2017.

Jackson, S.E. et al., 2015. Perceived weight discrimination in England: a population-based study of adults aged $\geqslant 50$ years. International Journal of Obesity (2005), 39(5), pp.858-864. Available at: http://www.ncbi.nlm.nih.gov/pmc/articles/PMC4309989/.

Keith, S.W. et al., 2011. Use of self-reported height and weight biases the body mass indexmortality association. International journal of obesity (2005), 35(3), pp.401-408. Available at: http://dx.doi.org/10.1038/ijo.2010.148. 
Kempen, G.I. et al., 1996. The assessment of ADL among frail elderly in an interview survey: self-report versus performance-based tests and determinants of discrepancies. The journals of gerontology. Series B, Psychological sciences and social sciences, 51(5), pp.P254-P260.

Kim, J.-M. et al., 2008. Predictive value of folate, vitamin B12 and homocysteine levels in latelife depression. The British journal of psychiatry: the journal of mental science, 192(4), pp.268-74. Available at: http://bjp.rcpsych.org/content/192/4/268 [Accessed April 13, 2016].

King, W.C. et al., 2013. Associations between physical activity and mental health among bariatric surgical candidates. Journal of psychosomatic research, 74(2), pp.161-169. Available at: http://www.ncbi.nlm.nih.gov/pmc/articles/PMC3556899/.

Kuczmarski, M.F., Kuczmarski, R.J. \& Najjar, M., 2001. Effects of Age on Validity of SelfReported Height, Weight, and Body Mass Index: Findings from the Third National Health and Nutrition Examination Survey, 1988-1994. Journal of the American Dietetic Association, 101(1), pp.28-34. Available at: http://www.sciencedirect.com/science/article/pii/S0002822301000086.

Marks, D.F., 2016. Dyshomeostasis, obesity, addiction and chronic stress. Health Psychology Open, 3(1), p.2055102916636907. Available at: http://hpo.sagepub.com/lookup/doi/10.1177/2055102916636907 [Accessed September 27, 2016].

Marks, D.F., 2015. Homeostatic theory of obesity. Health Psychology Open, 2(1), p.2055102915590692. Available at: http://hpo.sagepub.com/lookup/doi/10.1177/2055102915590692 [Accessed September 27, 2016].

Mendes De Leon, C.F., Rapp, S.S. \& Kasl, S. V., 1994. Financial strain and symptoms of depression in a community sample of elderly men and women: A longitudinal study. Journal of Aging and Health, 6(4), pp.448-468.

Ogden, C.L. et al., 2014. Prevalence of childhood and adult obesity in the United States, 20112012. Jama, 311(8), pp.806-814. Available at: http://www.ncbi.nlm.nih.gov/pubmed/24570244.

Palinkas, L.A., Wingard, D.L. \& Barrett-Connor, E., 1996. Depressive symptoms in overweight and obese older adults: A test of the "Jolly Fat" Hypothesis. Journal of Psychosomatic Research, 40(1), pp.59-66.

Pan, A. et al., 2012. Bidirectional association between depression and obesity in middle-aged and older women. International journal of obesity, 36(4), pp.595-602. Available at: http://www.nature.com.prxy4.ursus.maine.edu/ijo/journal/v36/n4/full/ijo2011111a.html [Accessed May 4, 2016].

Puhl, R.M., Andreyeva, T. \& Brownell, K.D., 2008. Perceptions of weight discrimination: prevalence and comparison to race and gender discrimination in America. Int J Obes, 32(6), pp.992-1000. Available at: http://dx.doi.org/10.1038/ijo.2008.22.

Robbins, M.A. et al., 1994. Unmedicated blood pressure levels and quality of life in elderly hypertensive women. Psychosomatic medicine, 56(3), pp.251-259. 
Roberts, R.E. et al., 2002. Are the fat more jolly? Annals of Behavioral Medicine, 24(3), pp.169180. Available at: http://dx.doi.org/10.1207/S15324796ABM2403_02.

Roberts, R.E. et al., 2000. Are the obese at greater risk for depression? American Journal of Epidemiology, 152(2), pp.163-170.

Roberts, R.E. et al., 2003. Prospective association between obesity and depression: evidence from the Alameda County Study. International journal of obesity and related metabolic disorders : journal of the International Association for the Study of Obesity, 27(4), pp.514521.

Roehling, M. V, Roehling, P. V \& Pichler, S., 2007. The relationship between body weight and perceived weight-related employment discrimination: The role of sex and race. Journal of Vocational Behavior, 71(2), pp.300-318. Available at: http://www.sciencedirect.com/science/article/pii/S0001879107000437.

St-Arnaud-McKenzie, D., Payette, H. \& Gray-Donald, K., 2010. Low physical function predicts either 2-year weight loss or weight gain in healthy community-dwelling older adults. The NuAge longitudinal study. Journals of Gerontology - Series A Biological Sciences and Medical Sciences, 65 A(12), pp.1362-1368.

Thurber, S., Snow, M. \& Honts, C.R., 2002. The Zung Self-Rating Depression Scale: convergent validity and diagnostic discrimination. Assessment, 9(4), pp.401-405.

Vogelzangs, N. et al., 2010. Obesity and onset of significant depressive symptoms: results from a prospective community-based cohort study of older men and women. The Journal of clinical psychiatry, 71(4), pp.391-9. Available at: http://www.psychiatrist.com/JCP/article/Pages/2010/v71n04/v71n0403.aspx [Accessed May 4, 2016].

de Wit, L. et al., 2010. Depression and obesity: a meta-analysis of community-based studies. Psychiatry research, 178(2), pp.230-5. Available at: http://www.sciencedirect.com/science/article/pii/S016517810900170X [Accessed May 27, 2015].

World Health Organization, 2010. Obesity. Available at: http://www.who.int/topics/obesity/en/.

Xiang, X. \& An, R., 2015. Obesity and onset of depression among U.S. middle-aged and older adults. Journal of psychosomatic research, 78(3), pp.242-8. Available at: http://www.sciencedirect.com/science/article/pii/S0022399914004449 [Accessed March 9, 2016].

Zung, W.K., 1965. A self-rating depression scale. Archives of General Psychiatry, 12(1), pp.6370. Available at: http://dx.doi.org/10.1001/archpsyc.1965.01720310065008. 


\begin{tabular}{|c|c|c|c|c|}
\hline Variable & Value & $\%$ Female & $\%$ Male & $p<.05$ \\
\hline \multirow[t]{4}{*}{ Age } & $50-59$ & 32.0 & 29.6 & \\
\hline & $60-69$ & 25.7 & 35.6 & \\
\hline & $70-79$ & 32.8 & 26.4 & \\
\hline & 80 or older & 9.5 & 8.4 & \\
\hline Years of Education $\geq 12$ & & 94.6 & 95.6 & \\
\hline Married/Cohabitating & & 54.5 & 79.6 & * \\
\hline Recent Life Events & & 76.8 & 66.4 & $*$ \\
\hline Social Activity & $\operatorname{Mean}(\mathrm{SD})$ & $21.7(5.3)$ & $20.3(4.8)$ & \\
\hline Moderate/ High Isolation & & 30.4 & 18.3 & $*$ \\
\hline \multirow[t]{4}{*}{ Zung Depression Inventory } & $<40$ & 43.8 & 47.8 & \\
\hline & $40-49$ & 31.8 & 34.9 & \\
\hline & $50-59$ & 13.7 & 18.3 & \\
\hline & $\geq 60$ & 6.1 & 3.6 & \\
\hline Obesity Rate (objective) & & 38.3 & 39.6 & \\
\hline Obesity Status (self-report) & & 29.0 & 29.5 & \\
\hline \multirow[t]{4}{*}{ Chronic Conditions } & 0 & 34.8 & 32.8 & \\
\hline & 1 & 19.8 & 57.8 & \\
\hline & 2 & 16.0 & 20.0 & \\
\hline & 3 or more & 29.4 & 22.4 & \\
\hline \multirow[t]{4}{*}{ Physical Deficits } & None & 53.6 & 60.4 & \\
\hline & Low & 15.7 & 16.8 & \\
\hline & Medium & 14.7 & 6.8 & $*$ \\
\hline & High & 16.0 & 16.0 & \\
\hline
\end{tabular}

$* p<.05$ refers to differences in category prevalence by gender

1 
Table 2. Regression coefficients showing relations between BMI and depressive Symptoms

Cross-sectional

$\underline{\text { Objective BMI }^{\mathrm{A}}}$

$\underline{b}$

Model 1

Model 2

Model 3

Model 2

Model 3

Model 2

Model 3
0.16

0.16

0.08

\section{$\underline{\text { Objective BMI }}$}

$$
\underline{b}
$$

0.23

0.20

\section{$\underline{\text { Objective BMI }}{ }^{\mathrm{E}}$}

$$
\underline{b}
$$

0.01

0.02

0.02

$\underline{\text { Self-Report BMI }}$

$\underline{B}$

0.17

0.15

0.06
.06
$0.06=.008$

$0.06=.005$

$0.06=.16$
$\underline{S E} \quad \underline{p-v a l u e}$

$0.06=.006$

$0.06=.11$

$0.06>.25$

\section{Longitudinal: BMI Predicting Depressive Symptoms}

Longitudinal: Depressive symptoms Predicting BMI
$\underline{S E}$

p-value

$0.05<.001$

$0.05<.001$

$0.05<.001$

$\underline{\text { Self-Report BMI }}$

\section{$\underline{\text { Self-Report BMI }}$}

$\underline{\text { SE }} \quad$ p-value

$0.06<.001$

$0.06<.001$

$0.06<.001$

$\begin{array}{lll}0.24 & 0.06 & <.001 \\ 0.24 & 0.06 & <.001 \\ 0.20 & 0.06 & <.001\end{array}$

$\begin{array}{ccccc}\underline{\boldsymbol{S E}} & \underline{\boldsymbol{p} \text {-value }} & \underline{\boldsymbol{B}} & \underline{\boldsymbol{S E}} & \underline{\boldsymbol{p} \text {-value }} \\ 0.01 & >.25 & -0.01 & 0.02 & >.25 \\ 0.01 & >.25 & <0.01 & 0.02 & >.25 \\ 0.01 & =0.20 & <0.01 & 0.02 & >.25\end{array}$

Model $1=$ age, sex, education, and marital status. Outcome variable adjusted at baseline if longitudinal model

Model $2=$ Model $1+$ recent life events, social isolation, and social activity

Model $3=$ Model $2+$ chronic illness and physical performance deficits

${ }^{\mathrm{A}} n=810 \quad{ }^{\mathrm{B}} n=761 \quad{ }^{\mathrm{C}} n=582 \quad \mathrm{D}_{n=544} \quad{ }^{\mathrm{E}} n=540 \quad{ }^{\mathrm{F}} n=495$ 